\title{
Cost-effectiveness of Latent Tuberculosis Infection Screening before Immigration to Low-Incidence Countries
}

\author{
Jonathon R. Campbell, James C. Johnston, Victoria J. Cook, \\ Mohsen Sadatsafavi, R. Kevin Elwood, Fawziah Marra
}

Prospective migrants to countries where the incidence of tuberculosis (TB) is low (low-incidence countries) receive TB screening; however, screening for latent TB infection (LTBI) before immigration is rare. We evaluated the cost-effectiveness of mandated and sponsored preimmigration LTBI screening for migrants to low-incidence countries. We used discrete event simulation to model preimmigration LTBI screening coupled with postarrival follow-up and treatment for those who test positive. Preimmigration interferon-gamma release assay screening and postarrival rifampin treatment was preferred in deterministic analysis. We calculated cost per quality-adjusted life-year gained for migrants from countries with different TB incidences. Our analysis provides evidence of the cost-effectiveness of preimmigration LTBI screening for migrants to low-incidence countries. Coupled with research on sustainability, acceptability, and program implementation, these results can inform policy decisions.

$\mathrm{T}$ he World Health Organization (WHO) has continued working toward tuberculosis (TB) elimination, aiming to reduce the overall TB burden by $\approx 90 \%$ to $<1$ case $/ 1$ million persons in countries where TB incidence is low (lowincidence countries) (1). Meeting this target will require new and innovative strategies. Typically, the TB burden in low-incidence countries is highest among populations born abroad; $\approx 70 \%$ of TB cases occur in these populations in Canada, the United States, and much of Europe (2). For the most part, TB prevention in these populations has focused on identifying persons with active TB before immigration to reduce transmission after arrival. Stagnant rates of TB suggest additional methods are required to accelerate declines in TB incidence (3).

Author affiliations: University of British Columbia, Vancouver, British Columbia, Canada (J.R. Campbell, J.C. Johnston,

V.J. Cook, M. Sadatsafavi, R.K. Elwood, F. Marra); British

Columbia Centre for Disease Control, Vancouver (J.C. Johnston,

V.J. Cook, R.K. Elwood)

DOI: https://doi.org/10.3201/eid2504.171630
Universal or targeted postarrival screening for latent TB infection (LTBI) has been suggested as a method to accelerate the decline of TB (4); however, domestic LTBI programs exhibit suboptimal performance (5), are resource intensive (6), and may not be cost-effective (7). One major reason for the reduced effectiveness of postarrival LTBI screening programs is the substantial attrition in the LTBI cascade of care. More than half of patients do not reach the point of initiating treatment, which results in fewer than one fifth completing treatment (5).

Currently, most immigrant-receiving, low-incidence countries employ mandatory preimmigration medical exams (8). As part of these medical exams, a chest radiograph and medical evaluation are performed to detect TB disease before arrival or identify those who may be at increased risk for TB disease in the future; these costs are borne by the patient within their country of origin. Only a select few countries employ some form of mandated LTBI screening (8), and data are scarce on the yield of such programs.

A report sponsored by the US Centers for Disease Control and Prevention (Atlanta, GA, USA) suggested mandatory LTBI screening and treatment as part of routine preimmigration medical exams (9); however, this strategy was viewed as inequitable and unjustly coercive (10) and has never been employed. Alternatively, mandating and fully sponsoring only LTBI screening as a formal part of the immigration process would avoid such ethics quandaries and could substantially reduce postarrival TB incidence. Preimmigration screening coupled with postarrival follow-up could improve the yield of LTBI screening programs $>2$-fold (5), because all case-patients reporting postarrival would already have completed LTBI screening.

We evaluated the cost-effectiveness of mandating and fully sponsoring LTBI screening in prospective migrants as part of routine preimmigration medical exams, coupled with passive postarrival follow-up and treatment. We evaluated 6 strategies among migrants from 4 different TB incidence groups to determine the optimal strategy in each group for this intervention. 


\section{Methods}

\section{Model Overview}

We chose discrete event simulation for this model because of its flexibility in varying transition times between health states in a single simulation, ability to simulate simultaneous events, and capability to model several different patient covariates. These advantages make it preferable to traditional Markov models and enable the creation of a highly representative cohort in a single simulation (11). We modeled new migrants, which in this evaluation refers specifically to persons who have been granted permanent resident status but have not yet become citizens of the countries they reside in. Of interest were migrants from countries belonging to 4 distinct TB incidence categories: low, $<30$ cases/100,000 persons/year; moderate, $\geq 30$ and $<100$ cases/100,000 persons/year; high, $\geq 100$ and $<200$ cases/100,000 persons/year; and very high, $\geq 200$ cases/100,000 persons/year.

We further defined the 4 populations of interest by 4 covariates: patient age, bacillus Calmette-Guérin (BCG) vaccination status, chest radiograph results, and LTBI prevalence. Patient age was defined based on an age distribution of a reference cohort of permanent residents to Canada in 2014 (12). BCG vaccination was determined through presence of a universal BCG vaccination policy in each country of origin and adjusted by 36-year average BCG vaccine uptake (13-15). For chest radiograph, a reference cohort of permanent residents who came to Ontario during 2002-2011 was used to identify prevalence of abnormal chest radiograph results (15). LTBI prevalence was calibrated in each population using 2-year TB incidence in permanent resident cohorts to Ontario during 2002-2011 (15) and age-adjusted using the results of a meta-analysis of test-positive rates (16).

We estimated LTBI prevalence using several assumptions. First, we assumed that $85 \%$ of incident TB resulted from reactivation of LTBI (17); second, that TB reactivation did not change over time post arrival (18); and last, that LTBI prevalence approximately matched reported rates of interferon-gamma release assay (IGRA) positivity in persons from each of the 4 TB incidence categories (16). In sum, an LTBI reactivation rate of 1.1 cases/1,000 personyears approximated literature values and yielded reasonable estimates of LTBI prevalence (17).

The model evaluates implementation of the intervention: preimmigration LTBI screening coupled with postarrival follow-up and treatment. The base case in this model was considered to be preimmigration TB screening without any evaluation for LTBI before or after arrival but with routine postarrival follow-up for those flagged through TB screening. We calibrated baseline TB incidence estimates and rates of postarrival follow-up to TB incidence data in permanent resident cohorts to Ontario during 2002-2011 (15). We considered 3 preimmigration LTBI screening options and 2 postarrival LTBI treatment options, for a total of 6 unique strategies to compare with the base case (Table 1).

We screened migrants with a tuberculin skin test (TST), IGRA, or sequential screening, in which persons testing positive by TST were given a confirmatory IGRA. We defined a positive TST result as an induration measuring $\geq 10 \mathrm{~mm}$ and a positive IGRA result using manufacturer's recommendations, with IGRA performance being a composite measure of results from commercially available products $(19-21)$. Although preimmigration testing was mandated, postarrival follow-up and treatment was not mandated and instead assumed to be passive, following published rates of postarrival follow-up in several countries (22). That is, in migrants who tested positive for LTBI, it was recommended that they attend a clinic for treatment postarrival, but no system was in place to enforce this. Those who reported for care postarrival would be treated with 9 months of isoniazid or 4 months of rifampin.

The model took a healthcare system perspective for the fully sponsored and mandated preimmigration LTBI screening: all LTBI screening costs preimmigration, along with typical postarrival costs, were the responsibility of the receiving country's healthcare system. We used a 3\% annual discount rate for costs and outcomes (23) and a 25-year time horizon from arrival. The main outcomes of the model were quality-adjusted life-years (QALYs), number of TB cases, and costs per 1,000 permanent residents from each of the 4 populations analyzed. These data were used to calculate the cost-effectiveness ratio, a measure that indicates the cost per additional QALY gained by an intervention strategy compared with the base case (Appendix, https://wwwnc.cdc.gov/EID/article/25/4/171630-App1.pdf).

A simplified model structure is displayed in Figure 1. In the intervention, migrants were given an LTBI diagnostic test along with the rest of their medical exam; those who tested positive were referred for postarrival followup. Those who complied with postarrival follow-up were recommended for LTBI therapy. After initiating treatment, they either completed treatment in full, partially completed treatment, or ceased due to an adverse event that may result in death. After treatment, results for all patients were simulated to the 25-year time horizon, with annual risks of TB reactivation and death.

We made the following assumptions in the model. Those with previous TB or an abnormal chest radiograph result identified during the preimmigration medical exam were also referred for postarrival follow-up. With the intervention, all those who began screening completed it, 
Table 1. Intervention strategies for screening and treatment of latent TB infection in immigrants*

\begin{tabular}{|c|c|}
\hline Intervention strategy & Preimmigration \\
\hline Base case & $\begin{array}{l}\text { TB screening as part of routine preimmigration medical } \\
\text { exams, consisting of a chest radiograph, medical history, } \\
\text { and symptom screen. If diagnosed with TB, treatment } \\
\text { must be completed before immigrating. }\end{array}$ \\
\hline TST/INH & $\begin{array}{c}\text { In addition to the base case, a TST is performed at the } \\
\text { time of the medical exam. If the result is positive (induration } \\
\geq 10 \mathrm{~mm} \text { ) referral is made for follow-up postarrival. } \\
\text { If the TST result is negative, no further action is taken. } \\
\text { Same as above. }\end{array}$ \\
\hline IGRA/INH & $\begin{array}{c}\text { In addition to the base case, an IGRA is placed at the time of the } \\
\text { medical exam. If the result is positive (as defined by the } \\
\text { manufacturer) referral is made for follow-up postarrival. If the } \\
\text { IGRA result is negative, no further action is taken. If the IGRA } \\
\text { result is indeterminate, a second is performed; a second } \\
\text { consecutive indeterminate is treated as a negative. } \\
\text { Same as above. }\end{array}$ \\
\hline SEQ/INH & $\begin{array}{l}\text { In addition to the base case, a TST is placed at the time of the } \\
\text { medical exam. If the result is positive (as defined by an } \\
\text { induration } \geq 10 \mathrm{~mm} \text { ) a second test is performed with an IGRA. } \\
\text { If the subsequent IGRA result is positive (as defined by the } \\
\text { manufacturer) referral is made for follow-up postarrival. } \\
\text { If the initial TST is negative or if the subsequent IGRA is } \\
\text { negative, no further action is taken. If the IGRA result is } \\
\text { indeterminate, a second is performed; a second } \\
\text { consecutive indeterminate is treated as a negative. }\end{array}$ \\
\hline & \\
\hline
\end{tabular}
Routine follow-up of those with abnormal
chest radiograph results or previous TB. xams, consisting of a chest radiograph, medical history, mptom screen. If diagnosed with TB treatment

Recommendation for follow-up; if patient reports for follow-up, 9-month course of INH.

Recommendation for follow-up; at follow-up, 4-month course of RIF.

Recommendation for follow-up; if patient reports for follow-up, 9-month course of INH. manufacturer) referral is made for follow-up postarrival. If the RA result is negative, no further action is taken. If the IGRA consecutive indeterminate is treated as a negative.
condeterminate, a second is performed; a second Same as above.

Recommendation for follow-up; if patient reports for follow-up, 4-month course of RIF. Recommendation for follow-up; at follow-up, 9-month course of INH. induration $>10 \mathrm{~mm}$ ) a second test is performed with an IGRA. If the subsequent IGRA result is positive (as defined by the manufacturer) referral is made for follow-up postarrival. the initial TST is negative or if the subsequent IGRA is indeterminate, a second is performed; a second Same as above.

Recommendation for follow-up; at follow-up, 4-month course of RIF. *No intervention required for migrants with negative results of base case screening. IGRA, interferon-gamma release assay; INH, isoniazid; RIF, rifampin; SEQ, sequential screening; TB, tuberculosis TST, tuberculin skin test.

eliminating dropout during this stage of the LTBI cascade of care. Drug-resistant TB and self-cure of LTBI were not modeled. It was assumed that all those who tested positive were offered LTBI treatment to limit extrapolation of care provider decisions. All reactivation TB cases had a $17.6 \%$ chance of causing a secondary case; further transmission was not modeled (Appendix). Modeling was completed in Simio version 8.146.14121 (Simio LLC, https://www.simio.com).

\section{Model Parameters}

We derived model estimates from the literature or expert opinion (Table 2). A meta-analysis provided evidence for domestic LTBI program performance (5), therapy efficacy was derived from the literature $(24,27,28)$, and adverse events were imputed from several randomized controlled trials reported in previous analysis $(24,25)$. Diagnostic performance of LTBI screening tests was derived from systematic reviews and modeled to be the same in each country (19-21). Adherence with postarrival follow-up was estimated by reanalysis of reported data (22) (Appendix Figure 1). Death from tuberculosis (3), probability of TB therapy extension (30), and relapse rate (31) were derived from Canada sources. Life tables for Canada estimated background mortality (32).
We derived all costs from Canada sources and assumed that the costs of screening abroad were equal to screening costs in Canada. We derived costs for LTBI treatment and screening, including drugs, screening tests, routine monitoring, and clinician time, from the British Columbia Centre for Disease Control. Adverse event costs, including hospitalization rates and time, and the cost of TB disease were as reported in the literature $(30,33,34)$. We inflated all costs to 2016 Canadian dollars using consumer price indices (35) (Table 3).

We derived health utility data from a study (38) in Canada of migrants who reported for postarrival follow-up. We based adjustments due to adverse events or hospitalization on previous studies $(30,33)$.

\section{Sensitivity Analysis}

We performed a probabilistic sensitivity analysis (PSA) to capture uncertainty of model estimates using an outer sample size of 1,000 and inner sample size of 50,000 (Tables $2,3)$. To guide policymakers, we created cost-effectiveness acceptability curves (CEAC) to determine the probability that the most cost-effective intervention strategy in deterministic analysis would fall below various willingness-topay (WTP) thresholds. Exploratory sensitivity analysis and additional probabilistic sensitivity analyses are included in the Appendix. 


\section{Results}

\section{Primary Results}

Among migrants from moderate- to very high-incidence countries, IGRA screening coupled with postarrival rifampin treatment was the optimal intervention strategy in deterministic analysis. Sequential screening coupled with postarrival rifampin treatment was the optimal intervention strategy among migrants from low-incidence countries. Intervention strategies involving TST identified the most migrants for postarrival follow-up, whereas strategies involving sequential screening identified the fewest. Intervention strategies involving rifampin resulted in the fewest TB cases ( $46 \%$ reduction compared with the base case) (Table 4$)$.

\section{Low-Incidence Countries}

For migrants from low-incidence countries, screening with TST alone resulted in a net loss in population QALYs because of poor specificity of the TST. Sequential screening, the most specific screening method, coupled with postarrival rifampin treatment yielded the lowest cost per QALY gained at $\$ 191,889$. IGRA screening, the most sensitive screening method, coupled with rifampin treatment resulted in the fewest TB cases (46.2\% reduction) but had a higher cost per QALY gained $(\$ 373,773)$ because of its lower specificity compared with that of sequential screening.

\section{Moderate-Incidence Countries}

For migrants from moderate-incidence countries, the optimal intervention strategy was IGRA screening coupled with postarrival rifampin treatment for those from moderate-incidence countries with a cost per QALY gained of $\$ 43,343$. Sequential screening coupled with postarrival rifampin treatment was cheaper overall but had a cost per QALY gained of $\$ 47,561$.

\section{High-Incidence Countries}

Among migrants from high-incidence countries, IGRA screening coupled with postarrival rifampin treatment was the optimal intervention strategy, at a cost per QALY

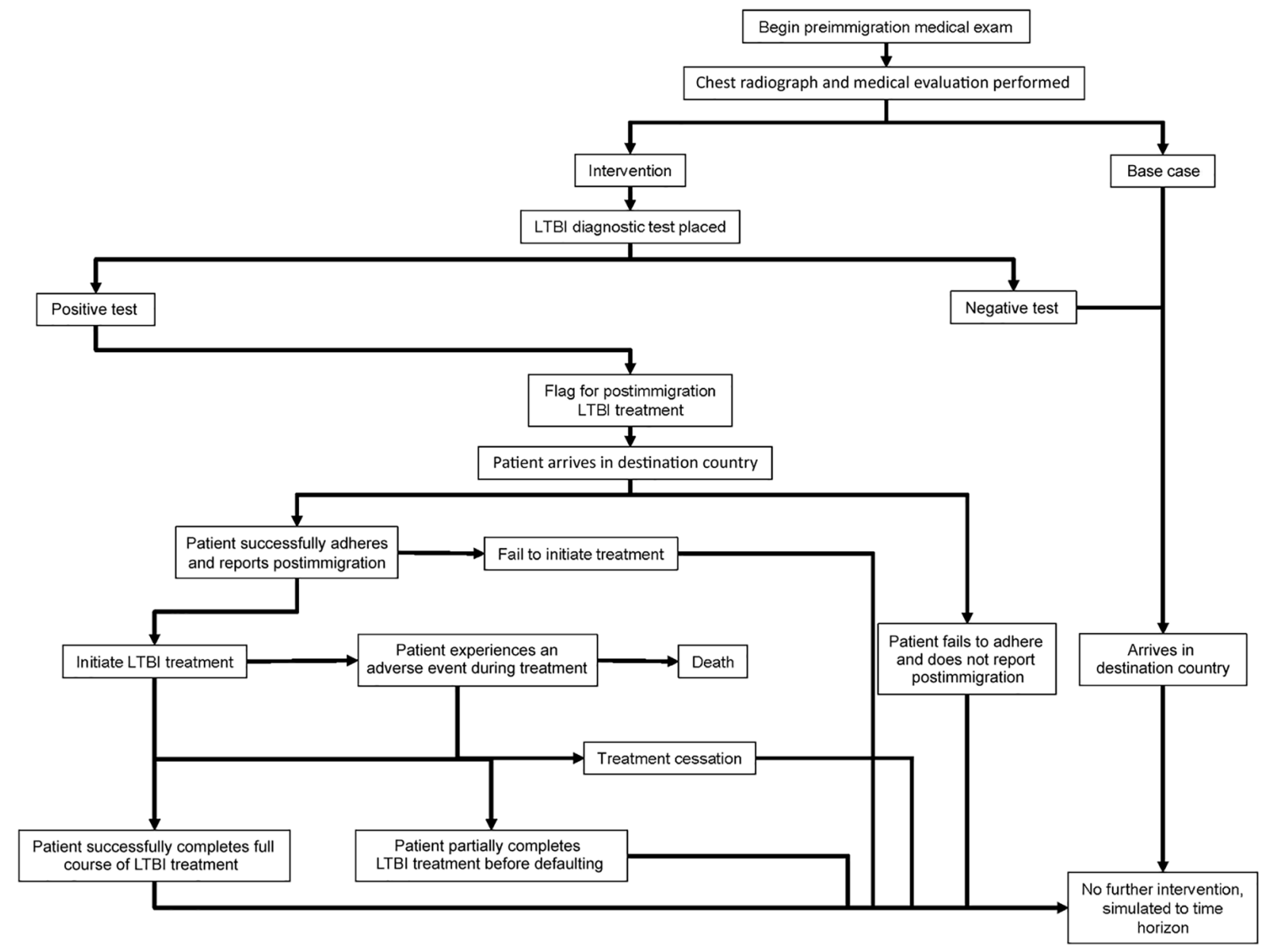

Figure 1. Flow structure of model used for cost-effectiveness analysis of screening and interventions of migrants for TB and LTBI. LTBI, latent tuberculosis infection; TB, tuberculosis. 
gained of $\$ 26,350$. Sequential screening coupled with rifampin treatment was less expensive, but also less efficient, with a cost per QALY gained of $\$ 29,997$.
Very High-Incidence Countries

Among migrants from very high-incidence countries, IGRA screening coupled with postarrival rifampin treatment had a

Table 2. Model parameter estimates and values used for sensitivity analyses of intervention strategies for screening and treatment of latent TB infection in immigrants*

\begin{tabular}{lcccc}
\hline Parameter & Estimate & $\begin{array}{c}\text { Range evaluated } \\
\text { in PSA }\end{array}$ & PSA distribution & References \\
\hline Screening parameters & & & & \\
$\quad$ TST sensitivity & 0.782 & $0.69-0.87$ & Beta $(43,12)$ & $(19)$ \\
TST specificity, no BCG & 0.974 & $0.963-0.982$ & Beta $(770,21)$ & $(20,21)$ \\
TST specificity, BCG & 0.602 & $0.561-0.642$ & Beta $(239,158)$ & $(20,21)$ \\
IGRA sensitivity & 0.889 & $0.688-0.993$ & Beta $(8,1)$ & $(19)$ \\
IGRA specificity & 0.957 & $0.946-0.968$ & Beta $(900,40)$ & $(20,21)$ \\
IGRA indeterminate $†$ & 0.06 & $0.05-0.07$ & Beta $(83,1286)$ & $(21)$ \\
Complete TST & 1 & Fixed & Fixed & \\
Complete medical evaluation§ & 1 & Fixed & Fixed &
\end{tabular}

Population characteristics $\mathbb{T}$

LTBI prevalence

Very high incidence $\quad 0.3162$

High incidence 0.2016

Moderate incidence 0.0902

Low incidence 0.0159

Abnormal chest radiograph results or previous TB

Very high incidence

High incidence $\quad 0.028$

Moderate incidence $\quad 0.029$

Low incidence $\quad 0.008$

Adherence to postarrival follow-up\#

Treatment parameters

Initiate**

Complete, INH

Complete, RIF

Adverse event, INH

Adverse event, RIF

Adverse event hospitalization

Death, INH

$\mathrm{LTBI}$ risk reduction, INH

$\mathrm{LTBI}$ risk reduction, $\mathrm{RIF}$

Partial risk reduction, INH

0.684

0.938

0.616

0.814

0.049

0.021

0.01

0.00000988

0.90

0.90

0.346

0.30

$7 d$

Adverse event duration

\section{TB parameters}

Death from TB

Reactivation rate

Abnormal CXR risk change

Extended therapy

Relapse rate

Hospitalization duration

Model parameters

BCG vaccination, $<30$ cases

$B C G$ vaccination, $\geq 30$ cases

$B C G$ vaccination uptake

Discount rate

Time horizon

$0.2686-0.3880$

$0.1706-0.2464$

$0.0763-0.1102$

$0.0135-0.0195$

Varied with reactivation rate

Varied with reactivation rate

Varied with reactivation rate

Varied with reactivation rate

$(12,15-17)$

$(12,15-17)$

$(12,15-17)$

$(12,15-17)$

$\begin{array}{ll}\text { Fixed } & \text { Fixed } \\ \text { Fixed } & \text { Fixed } \\ \text { Fixed } & \text { Fixed } \\ \text { Fixed } & \text { Fixed }\end{array}$

0.646-0.721

Beta $(404.50,186.87)$

(15)

(15)

(15)

(22)

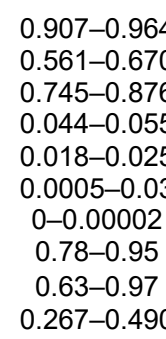

Beta $(180.83,11.95)$

Beta $(131.66,82.07)$

Beta $(249,4789)$

Beta $(109,4877)$

Beta $(1,99)$

$(24,25)$

$(24,25)$

Beta $(2,202495)$

(25)

(26)

Normal $(-2.3,0.5) \dagger \dagger$

Normal $(-2.3,0.8) \dagger \dagger$

Combination of normal

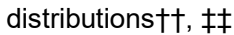

Normal $(-0.35,0.1) \dagger \dagger$

$(28,24)$

Expert opinion, (25)

Expert opinion

$(24,28)$

Gamma $(0.7,10)$

Expert opinion, (25)

$\begin{array}{ccc}0.0476 & 0.0391-0.0566 & \text { Beta }(76,1523) \\ 0.0011 & 0.0009-0.0013 & \text { Beta }(90.92,82545.55) \\ 3.9 & 3.0-4.9 & \text { Normal }(1.36,0.15) \dagger \dagger \\ 0.124 & 0.029-0.264 & \text { Beta }(2.366,16.713) \\ 0.0359 & 0.0197-0.0654 & \text { Normal }(-3.327,0.365) \dagger \dagger \\ 17 \mathrm{~d} & \text { Fixed } & \text { Fixed }\end{array}$

(15-17)

(29)

Expert opinion, (30)

(30)

Expert opinion, (30)

17

$\begin{array}{ccc}0.605 & 0.60-0.61 & \text { Beta }(45137,29502) \\ 0.998 & 0.997-0.999 & \text { Beta }(185381,384) \\ 0.837 & \text { Fixed } & \text { Fixed } \\ 0.03 & \text { Fixed } & \text { Fixed } \\ 25 y & \text { Fixed } & \text { Fixed }\end{array}$

$(12,13)$

$(12,13)$

(14)

(23)

NA

${ }^{*} \mathrm{AE}$, adverse event; BCG, bacillus Calmette-Guérin; IGRA, interferon-gamma release assay; INH, isoniazid; LTBI, latent tuberculosis infection; NA, not available; PSA, probabilistic sensitivity analysis; RIF, rifampin; TST, tuberculin skin test; TB, tuberculosis.

$\dagger$ Treated as a negative result if it occurred; was equally likely to occur in those with and without LTBI.

fWithout being mandatory, this value is $63.5 \%$ (imputed from $43.4 \%$ completing screening when $68.4 \%$ adhere with a follow-up appointment) (5).

$\S$ Without being mandatory, this value is $78 \%$ (imputed from 43.7 of 56 individuals completing medical evaluation) (5).

TVery high incidence, $>200$ cases/100,000; high incidence, $>100$ and $<200$ cases/100,000; moderate incidence, $>30$ and $<100$ cases/100,000; low incidence, $<30$ cases $/ 100,000$.

\#From a meta-analysis (22); see also Appendix (https://wwwnc.cdc.gov/EID/article/25/4/17-1630-App1.pdf).

**This model assumes all who report postarrival due to a positive preimmigration LTBI diagnostic test are offered treatment. Exploratory analysis adjusts this assumption so that only the number who would complete TST screening begin treatment.

t†Results from this distribution are exponentiated.

ł¥Formula: $0.33 \times(\operatorname{Normal}(-1.168,0.228))+0.374 \times(\operatorname{Normal}(-0.381,0.169))+0.293 \times 1$. 
cost per QALY gained of $\$ 16,291$ compared with the base case. Sequential screening with rifampin treatment again was slightly cheaper, resulting in a cost per QALY gained of $\$ 20,165$.

\section{Sensitivity Analysis}

Among migrants from low-incidence countries, sequential screening coupled with postarrival rifampin treatment was the most cost-effective option in deterministic analysis. In PSA, this intervention had a probability of cost-effectiveness of $49.1 \%$ at a WTP threshold of $\$ 50,000 /$ QALY and $50.7 \%$ at a WTP threshold of $\$ 100,000 /$ QALY. This probability did not substantially increase past these thresholds, however, resulting in a probability of cost-effectiveness of $52 \%$ at a WTP threshold of $\$ 200,000 /$ QALY (Figure 2, panel A).

Among migrants from moderate-, high-, and very highincidence countries, IGRA screening coupled with postarrival rifampin treatment was the most cost-effective option in deterministic analysis. This intervention strategy at WTP thresholds of $\$ 50,000 / \mathrm{QALY}$ gained had probabilities of cost-effectiveness of $57.5 \%$ among migrants from moderate-incidence countries (Figure 2, panel B), 68.2\% among migrants from high-incidence countries (Figure 2 , panel $\mathrm{C}$ ), and of $73.2 \%$ among migrants from very high-incidence countries (Figure 2, panel D). At a WTP threshold of $\$ 100,000 /$ QALY gained probabilities of costeffectiveness were $59.8 \%$ among migrants from moderateincidence countries, $70.6 \%$ among migrants from highincidence countries, and $75.2 \%$ among migrants from very high-incidence countries.

\section{Discussion}

The intervention of preimmigration LTBI screening followed by postarrival treatment among new migrants from countries with a TB incidence $\geq 30$ cases $/ 100,000$ persons appears to be an effective method for reducing TB incidence post-arrival. The use of IGRA screening coupled with postarrival rifampin treatment provided the lowest cost-effectiveness ratio in migrants from these countries. This intervention strategy reduced TB incidence by $>45 \%$ and yielded costs $<\$ 50,000 /$ QALY gained.

Table 3. Cost and QALY estimates and values used for sensitivity analysis of intervention strategies for screening and treatment of latent TB infection in immigrants*

\begin{tabular}{|c|c|c|c|c|}
\hline \multicolumn{5}{|c|}{ Range evaluated in } \\
\hline Parameter & Estimate, $\$$ & PSA & PSA distribution & References \\
\hline \multicolumn{5}{|l|}{ Costs } \\
\hline Full INH treatment & 992 & $804-1,179$ & Triangular, 804-1,179 & BCCDC, $(33,36)$ \\
\hline Drug costs & 181 & & & \\
\hline Nurse and clinician costs & 741 & & & \\
\hline Follow-up chest radiograph & 42 & & & \\
\hline Routine tests & 28 & & & \\
\hline Full RIF treatment & 575 & $464-686$ & Triangular, 464-686 & BCCDC, $(33,36)$ \\
\hline Drug costs & 98 & & & \\
\hline Nurse and clinician costs & 421 & & & \\
\hline Follow-up chest radiograph & 42 & & & \\
\hline Routine tests & 14 & & & \\
\hline Partial INH & 462 & 174-804 & Triangular, 174-804 & BCCDC, $(33,36)$ \\
\hline Partial RIF & 319 & $178-464$ & Triangular, 178-464 & BCCDC, $(33,36)$ \\
\hline Complete TST & 31 & $24-38$ & Triangular, 24-38 & BCCDC, $(33,36)$ \\
\hline TST cost & 11 & & & \\
\hline Nurse costs (2 visits) & 20 & & & \\
\hline Incomplete TST & 21 & $17-25$ & Triangular, 17-25 & BCCDC, $(33,36)$ \\
\hline IGRA & 54 & $31-62$ & Triangular, 31-62 & BCCDC, $(33,36)$ \\
\hline Kit and technician cost & 47 & & & \\
\hline Nurse costs & 7 & & & \\
\hline Chest radiograph & 42 & $32-52$ & Triangular, 32-52 & BCCDC, $(33,36)$ \\
\hline Cost per radiograph & 35 & & & \\
\hline Nurse costs & 7 & & & \\
\hline TB & 20,532 & $7,141-39,525$ & Gamma $(4.1064,5,000)$ & Expert opinion, $(33,34)$ \\
\hline LTBI adverse event & 732 & 549-916 & Triangular, 549-916 & (33) \\
\hline Hospitalization & 6,641 & 5,305-9,985 & Triangular, 5,305-9,985 & (30) \\
\hline Death & 26,933 & $13,079-40,788$ & Triangular, $13,079-40,788$ & (37) \\
\hline \multicolumn{5}{|l|}{ QALYs } \\
\hline LTBI & 0.81 & & Assumed & $(38)$ \\
\hline Healthy & 0.81 & $0.58-0.97$ & Beta $(7.85,1.84)$ & (38) \\
\hline Adverse event disutility & 0.2 & $0.15-0.25$ & Triangular, $\pm 25 \%$ & $(30,33)$ \\
\hline $\mathrm{TB}$ & 0.69 & $0.08-0.24 \dagger$ & Beta $(9,51)$ & $(38)$ \\
\hline Hospitalization & 0.5 & $0.28-0.51 \dagger$ & Beta $(19.5,30.5)$ & $(30)$ \\
\hline Death & 0 & Fixed & Fixed & Standard \\
\hline
\end{tabular}

${ }^{*}$ All costs are in 2016 Can \$. BCCDC, British Columbia Centre for Disease Control; IGRA, interferon-gamma release assay; INH, isoniazid; LTBI, latent tuberculosis infection; PSA, probabilistic sensitivity analysis; RIF, rifampin; TB, tuberculosis; TST, tuberculin skin test.

†Sampled as a percent decrement compared to healthy QALY. 
Because prevalence of LTBI was low among migrants from countries with a TB incidence $<30$ cases $/ 100,000$ persons and specificities of LTBI diagnostic tests are imperfect, this intervention may result in a high number of uninfected persons receiving treatment unnecessarily. This finding suggests that with some strategies, the QALYs lost due to treatment side effects among those with falsepositive diagnostic results may be greater than the QALYs gained by averted TB in those with true-positive diagnostic results. If screening and treatment must be performed in these low LTBI prevalence populations, more specific screening methods (i.e., sequential screening) are preferred to avoid inappropriate treatment.

Probabilistic sensitivity analysis suggests a certain degree of uncertainty in results. The behavior of CEACs as WTP thresholds increase suggests that the intervention offers small increases in population QALYs or large increases in cost in many replications. It is important to understand how well the model parameters represent the local setting when using the results of this analysis to inform evidence-based policy. These results suggest that intervention offers domestic benefits to the receiving country, but several factors need to be carefully examined. IGRA use in high-resource settings suffers from variability, in part related to several operational issues (39), and TST variability remains an issue (40). For both types of test, variability may be exacerbated in low-resource settings where LTBI prevalence rates are likely to be higher. In this model, we did not consider the costs of program initiation and maintenance; although they are outside the scope of this analysis, these costs merit careful evaluation when seeking to implement policy.

This model considered only the costs of persons who became permanent residents. The data from Canada indicated that $\approx 50 \%-60 \%$ of those who begin the process of becoming a permanent resident successfully complete it $(3,15)$. For migrants from very high-incidence countries, assuming only half of migrants receiving preimmigration

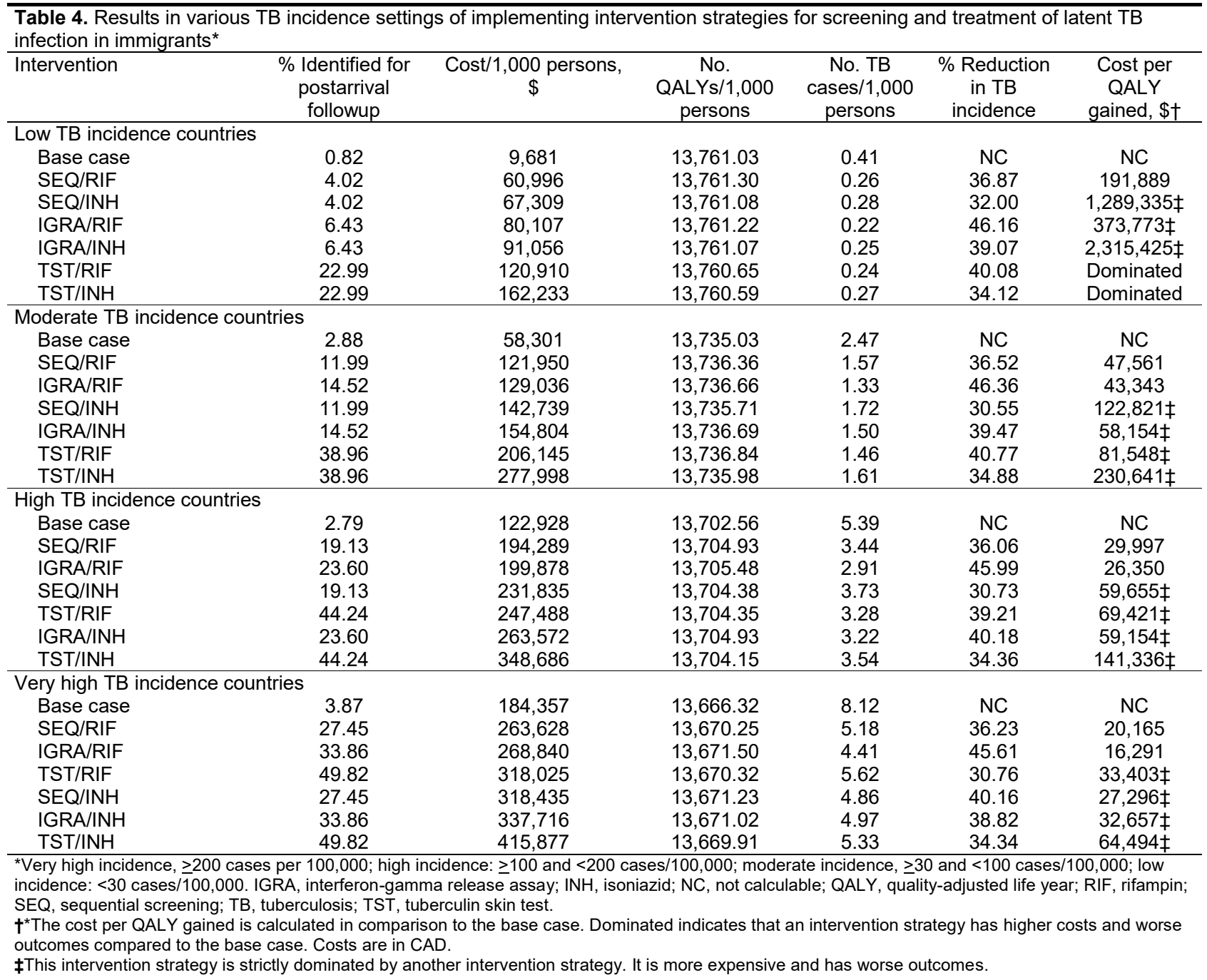



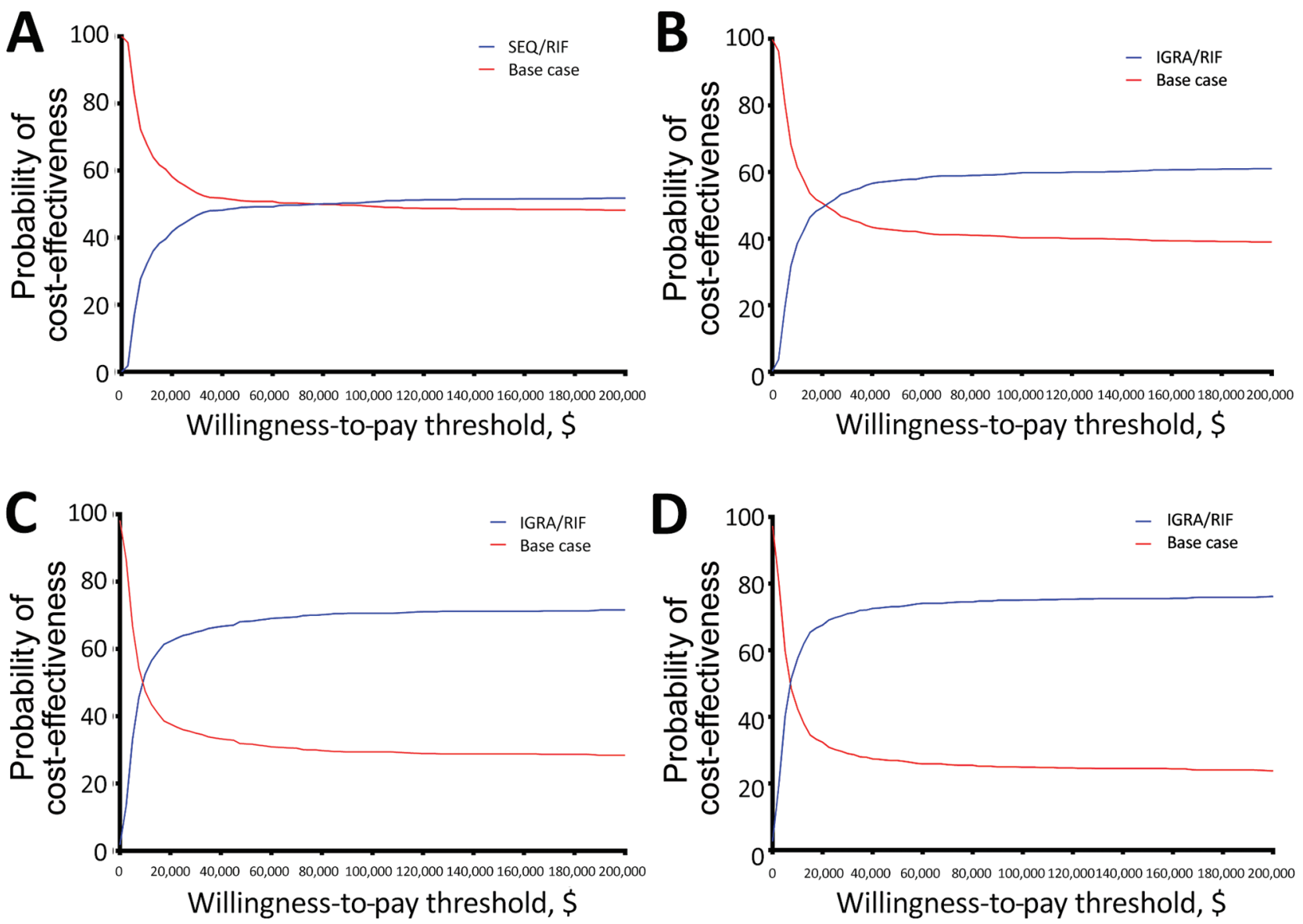

Figure 2. Cost-effectiveness acceptability curves of the base case of no intervention compared with intervention strategies in evaluation of screening and treatment of latent tuberculosis infection in immigrants. The graphs demonstrate the probability that an option is more cost-effective at various willingness-to-pay thresholds per quality adjusted life year gained. A) Comparison of the base case with the intervention strategy of preimmigration SEQ screening coupled with postarrival RIF treatment among migrants from low-incidence countries. B) Comparison of the base case with the intervention strategy of preimmigration IGRA screening coupled with postarrival rifampin treatment among migrants from moderate-incidence countries. C) Comparison of the base case with the intervention strategy of preimmigration IGRA screening coupled with postarrival RIF treatment among migrants from high-incidence countries. D) Comparison of the base case with the intervention strategy of preimmigration IGRA screening coupled with postarrival RIF treatment among migrants from very high-incidence countries. IGRA, interferon-gamma release assay; RIF, rifampin; SEQ, sequential.

screening became permanent residents, the cost-effectiveness ratio increased $60 \%$ to $\approx \$ 26,000$ when the intervention strategy was IGRA coupled with rifampin. Another consideration is the feasibility of the intervention. In a country like Canada, $2 \%-3 \%$ of new permanent residents are requested to follow up postarrival based on preimmigration medical exams $(3,15)$. If the country implemented preimmigration IGRA screening for migrants from moderate- to very high-incidence countries, $17.6 \%$ would be requested to follow up postarrival $(3,15)$. However, coupling IGRA with postarrival rifampin treatment could prevent $3.9 \%$ of all TB cases in Canada in the first year $(3,12,15)$. Applied to new permanent residents to Canada in 2014, this process would increase the number requested to follow up postarrival from 6,100 to 45,800 but would result in the prevention of $61 \mathrm{~TB}$ cases in the first year (1 case prevented/
651 additional postarrival referrals). If this process were then consistently implemented in successive cohorts in the future, it could annually prevent $\approx 400$ TB cases.

Regardless of how preimmigration LTBI screening is implemented, investment in LTBI infrastructure in high TB incidence settings will be essential for global TB elimination. Evidence suggests that introduction of routine preimmigration TB screening in many high-income, low-incidence countries has played a role in improving infrastructure for TB programs in low-resource areas (41). Further introducing LTBI screening as part of these routine medical exams may have similar impact.

The cost-effectiveness of preimmigration LTBI screening and postarrival treatment has not been evaluated since 2003. Previously, Schwartzman and Menzies (42) examined the idea of preimmigration TST screening in addition to 
standard preimmigration chest radiograph coupled with postarrival isoniazid treatment. They found the cost per TB case prevented was approximately Can $\$ 94,500$. In our study, using this intervention strategy in very high incidence countries resulted in a cost per TB case prevented of approximately Can $\$ 83,000$. Schwartzman et al. (43) later investigated the cost associated with performing a TST in all new legal immigrants from Mexico, a low-incidence country, and coupling it with postarrival isoniazid treatment. This resulted in a cost per TB case prevented of $\$ 1.2$ million (2016 Can \$). Using this same intervention strategy in our study resulted in a cost per TB case prevented of \$1.1 million (2016 Can \$). By evaluating new strategies applied to a variety of TB incidence settings, our study represents a much-needed update to the literature.

Our analysis has several strengths. Use of discrete event simulation enabled realistic modeling of time spent in various health states, which is difficult to implement in Markov models. This type of model also allowed agerepresentative modeling of new migrants for application of age-adjusted LTBI prevalence. The source of most of the cost data was the British Columbia Center for Disease Control, which handles most TB cases in the province of British Columbia. This analysis estimated LTBI prevalence and abnormal chest radiograph prevalence using several years of immigration and TB data from Ontario. The data are likely to be generalizable, because Ontario accepts $40 \%$ of new permanent residents (12) and the data fit well with reported LTBI prevalence estimates (16), suggesting these parameters are reflective of long-term TB trends.

In this study, we assumed that all migrants were recommended postarrival LTBI treatment when they had a positive LTBI diagnostic test, which is not necessarily true; for some persons, the risk for serious adverse events may outweigh the benefit of treatment. Social factors and concurrent conditions may increase the risk for reactivation of LTBI. We have shown that the benefits of rifampin treatment for migrants from moderate- to very high-incidence countries who test positive by IGRA preimmigration outweigh the potential risks of adverse events. However, in practice, individual adverse-event risk is considered, and treatment may not be offered to all migrants. Further research designed to identify the specific populations who should be offered treatment would help inform future analyses.

We derived the reactivation rate of LTBI from the literature, but because many of those studies were based on TB incidence in those who were positive by TST, it is possible that the predictive value of the TST caused underestimation of true reactivation rates. Our analysis did not consider 3 months of once-weekly isoniazid and rifapentine as an LTBI treatment modality because it was not universally available. Literature data, however, suggest this modality may yield similar results to rifampin treatment (44).
Our analysis used a healthcare system perspective, which does not consider costs incurred by persons experiencing the intervention (45). It is possible that consideration of costs and benefits from a societal perspective would change the results of this analysis; however, it is also likely that this difference would strengthen the preference for screening with IGRA, which requires only 1 visit, instead of TST, which requires 2 , due to reduced absenteeism associated with IGRA testing. Costs per QALY gained may increase for all strategies if the time costs for migrants to follow up for LTBI treatment were considered. Finally, we assumed that TB reactivation was constant, which, while demonstrated previously (18), contradicts the common paradigm of decreasing risk over time (46). Where possible, we performed sensitivity analyses to view the effects our limitations may have on our results to better inform decision makers.

In conclusion, preimmigration IGRA screening coupled with postarrival rifampin treatment among migrants from countries with moderate to very high incidence of TB resulted in the lowest cost-effectiveness ratios. This evidence can be used to support policy decisions surrounding preimmigration LTBI screening in high-income, immigrant-receiving countries, when coupled with evaluations on program implementation, acceptability, and sustainability. Next steps in research should be to identify subgroups at highest risk for progression to $\mathrm{TB}$ disease to limit individual risk associated with LTBI treatment and improve the likelihood of feasibility and sustainability.

\section{Acknowledgments}

The authors would like to thank Shannon Kopp and John Darras for their help with costing and Simio LLC (http://www.simio.com) for allowing use of their software and replication runner.

J.C.J. and M.S. have received funding from the Michael Smith Foundation for Health Research. M.S. also received salary support from the Canadian Institutes of Health Research.

J.R.C., J.C.J., and F.M. were involved in development of the study objective and design. J.R.C. performed data collection, created the model, performed data analysis and interpretation, and drafted the manuscript. J.C.J. provided expert input on the data informing the model, data interpretation, and performed manuscript editing. M.S. reviewed the model for errors, provided expert input for data analysis, and edited the manuscript. V.J.C. provided expert input on the data informing the model and edited the manuscript. R.K.E. provided expert input on the data interpretation and edited the manuscript. F.M. reviewed the model inputs collected and the data analysis, provided expert input for data interpretation, and edited the manuscript. All authors approved the study objective, design, and final manuscript. 


\section{About the Author}

Dr. Campbell received his PhD from the University of British Columbia in Vancouver and is currently a postdoctoral fellow at McGill University in Montreal, Quebec, Canada. His primary research interests include health economics, evidence-based public health policy, and infectious disease.

\section{References}

1. World Health Organization. Framework towards tuberculosis elimination in low-incidence countries. Geneva: The Organization; 2014 [cited 2018 Mar 7]. http://www.who.int/tb/publications/ elimination_framework/en/

2. Pareek M, Greenaway C, Noori T, Munoz J, Zenner D. The impact of migration on tuberculosis epidemiology and control in high-income countries: a review. BMC Med. 2016;14:48. http://dx.doi.org/10.1186/s12916-016-0595-5

3. Public Health Agency of Canada. Canadian tuberculosis standards, 7th ed. Ottawa (ON): Government of Canada; 2014 [cited 2018 Mar 7]. https://www.canada.ca/en/public-health/services/infectiousdiseases/canadian-tuberculosis-standards-7th-edition.html

4. Taylor Z, Nolan CM, Blumberg HM; American Thoracic Society; Centers for Disease Control and Prevention; Infectious Diseases Society of America. Controlling tuberculosis in the United States. Recommendations from the American Thoracic Society, CDC, and the Infectious Diseases Society of America. MMWR Recomm Rep. 2005;54(RR-12):1-81.

5. Alsdurf H, Hill PC, Matteelli A, Getahun H, Menzies D. The cascade of care in diagnosis and treatment of latent tuberculosis infection: a systematic review and meta-analysis. Lancet Infect Dis. 2016;16:1269-78. http://dx.doi.org/10.1016/S1473-3099(16) 30216-X

6. Campbell J, Marra F, Cook V, Johnston J. Screening immigrants for latent tuberculosis: do we have the resources? CMAJ. 2014;186:246-7. http://dx.doi.org/10.1503/cmaj.131025

7. Campbell JR, Sasitharan T, Marra F. A systematic review of studies evaluating the cost utility of screening high-risk populations for latent tuberculosis infection. Appl Health Econ Health Policy. 2015;13:325-40. http://dx.doi.org/10.1007/s40258-015-0183-4

8. Pareek M, Baussano I, Abubakar I, Dye C, Lalvani A. Evaluation of immigrant tuberculosis screening in industrialized countries. Emerg Infect Dis. 2012;18:1422-9. http://dx.doi.org/10.3201/ eid1809.120128

9. Institute of Medicine (US) Committee on the Elimination of Tuberculosis in the United States. Ending neglect: the elimination of tuberculosis in the United States. Geiter L, editor. Washington (DC): National Academies Press; 2000.

10. Coker R, van Weezenbeek KL. Mandatory screening and treatment of immigrants for latent tuberculosis in the USA: just restraint? Lancet Infect Dis. 2001;1:270-6. http://dx.doi.org/10.1016/ S1473-3099(01)00122-0

11. Karnon J, Stahl J, Brennan A, Caro JJ, Mar J, Möller J; ISPOR-SMDM Modeling Good Research Practices Task Force. Modeling using discrete event simulation: a report of the ISPOR-SMDM Modeling Good Research Practices Task Force- 4. Value Health. 2012;15:821-7. http://dx.doi.org/10.1016/j. jval.2012.04.013

12. Statistics Canada. Report on the demographic situation in Canada: permanent and temporary immigration to Canada from 2012 to 2014. Ottawa (ON): Government of Canada; 2016 [cited 2018 Mar 7]. http://www.statcan.gc.ca/pub/91-209-x/2016001/article/ 14615-eng.htm

13. Badar S, Araújo T, Zwerling A, Pai M. BCG world atlas. 2nd edition. 2017 [cited 2018 Mar 7]. http://www.bcgatlas.org/index.php

14. World Health Organization; UNICEF. WHO-UNICEF estimates of BCG coverage. Geneva: World Health Organization; 2017 [cited
2018 Mar 7]. http://apps.who.int/immunization_monitoring/ globalsummary/timeseries/tswucoveragebcg.html

15. Khan K, Hirji MM, Miniota J, Hu W, Wang J, Gardam M, et al. Domestic impact of tuberculosis screening among new immigrants to Ontario, Canada. CMAJ. 2015;187:E473-81. http://dx.doi.org/ 10.1503/cmaj.150011

16. Campbell JR, Chen W, Johnston J, Cook V, Elwood K, Krot J, et al. Latent tuberculosis infection screening in immigrants to lowincidence countries: a meta-analysis. Mol Diagn Ther. 2015;19:107-17. http://dx.doi.org/10.1007/s40291-015-0135-6

17. Shea KM, Kammerer JS, Winston CA, Navin TR, Horsburgh CR Jr. Estimated rate of reactivation of latent tuberculosis infection in the United States, overall and by population subgroup.

Am J Epidemiol. 2014;179:216-25. http://dx.doi.org/10.1093/aje/ kwt246

18. Walter ND, Painter J, Parker M, Lowenthal P, Flood J, Fu Y, et al.; Tuberculosis Epidemiologic Studies Consortium. Persistent latent tuberculosis reactivation risk in United States immigrants. Am J Respir Crit Care Med. 2014;189:88-95.

19. Campbell JR, Krot J, Elwood K, Cook V, Marra F. A systematic review on TST and IGRA tests used for diagnosis of LTBI in immigrants. Mol Diagn Ther. 2015;19:9-24. http://dx.doi.org/ 10.1007/s40291-014-0125-0

20. Menzies D, Pai M, Comstock G. Meta-analysis: new tests for the diagnosis of latent tuberculosis infection: areas of uncertainty and recommendations for research. Ann Intern Med. 2007;146:340-54. http://dx.doi.org/10.7326/0003-4819-146-5-200703060-00006

21. Pai M, Zwerling A, Menzies D. Systematic review: T-cell-based assays for the diagnosis of latent tuberculosis infection: an update. Ann Intern Med. 2008;149:177-84. http://dx.doi.org/10.7326/ 0003-4819-149-3-200808050-00241

22. Chan IHY, Kaushik N, Dobler CC. Post-migration follow-up of migrants identified to be at increased risk of developing tuberculosis at pre-migration screening: a systematic review and meta-analysis. Lancet Infect Dis. 2017;17:770-9. http://dx.doi.org/ 10.1016/S1473-3099(17)30194-9

23. Sanders GD, Neumann PJ, Basu A, Brock DW, Feeny D, Krahn M, et al. Recommendations for conduct, methodological practices, and reporting of cost-effectiveness analyses. JAMA. 2016;316:1093103. http://dx.doi.org/10.1001/jama.2016.12195

24. Menzies D, Adjobimey M, Ruslami R, Trajman A, Sow O, Kim H, et al. Four months of rifampin or nine months of isoniazid for latent tuberculosis in adults. N Engl J Med. 2018;379:440-53. http://dx.doi.org/10.1056/NEJMoa1714283

25. Campbell JR, Johnston JC, Sadatsafavi M, Cook VJ, Elwood RK, Marra F. Cost-effectiveness of post-landing latent tuberculosis infection control strategies in new migrants to Canada. PLoS One. 2017;12:e0186778. http://dx.doi.org/10.1371/journal.pone.0186778

26. Salpeter SR. Fatal isoniazid-induced hepatitis. Its risk during chemoprophylaxis. West J Med. 1993;159:560-4.

27. International Union Against Tuberculosis Committee on Prophylaxis. Efficacy of various durations of isoniazid preventive therapy for tuberculosis: five years of follow-up in the IUAT trial. Bull World Health Organ. 1982;60:555-64.

28. Reichman LB, Lardizabal A, Hayden CH. Considering the role of four months of rifampin in the treatment of latent tuberculosis infection. Am J Respir Crit Care Med. 2004;170:832-5. http://dx.doi.org/10.1164/rccm.200405-584PP

29. Aldridge RW, Zenner D, White PJ, Williamson EJ, Muzyamba MC, Dhavan P, et al. Tuberculosis in migrants moving from high-incidence to low-incidence countries: a population-based cohort study of 519,955 migrants screened before entry to England, Wales, and Northern Ireland. Lancet. 2016;388:2510-8. http://dx.doi.org/10.1016/S0140-6736(16)31008-X

30. Holland DP, Sanders GD, Hamilton CD, Stout JE. Costs and cost-effectiveness of four treatment regimens for latent tuberculosis 
infection. Am J Respir Crit Care Med. 2009;179:1055-60. http://dx.doi.org/10.1164/rccm.200901-0153OC

31. Jasmer RM, Bozeman L, Schwartzman K, Cave MD, Saukkonen JJ, Metchock B, et al.; Tuberculosis Trials Consortium. Recurrent tuberculosis in the United States and Canada. Am J Respir Crit Care Med. 2004;170:1360-6. http://dx.doi.org/10.1164/ rccm.200408-10810C

32. Statistics Canada. Life tables, Canada, provinces, and territories. 2016 [cited 2018 Mar 7]. http://www5.statcan.gc.ca/olc-cel/ olc. action?objId $=84-537-X \&$ objType $=2 \&$ lang $=$ en $\&$ limit $=0$

33. Marra F, Marra CA, Sadatsafavi M, Morán-Mendoza O, Cook V, Elwood RK, et al. Cost-effectiveness of a new interferon-based blood assay, QuantiFERON-TB Gold, in screening tuberculosis contacts. Int J Tuberc Lung Dis. 2008;12:1414-24.

34. Menzies D, Lewis M, Oxlade O. Costs for tuberculosis care in Canada. Can J Public Health. 2008;99:391-6.

35. Statistics Canada. Consumer price index, annual average, not seasonally adjusted. 2019 [cited 2018 Mar 7].

https://www150.statcan.gc.ca/t1/tbl1/en/tv.action?pid=1810000501

36. Tan MC, Marra CA, Sadatsafavi M, Marra F, Morán-Mendoza O, Moadebi S, et al. Cost-effectiveness of LTBI treatment for TB contacts in British Columbia. Value Health. 2008;11:842-52. http://dx.doi.org/10.1111/j.1524-4733.2008.00334.x

37. Fassbender K, Fainsinger RL, Carson M, Finegan BA. Cost trajectories at the end of life: the Canadian experience. J Pain Symptom Manage. 2009;38:75-80. http://dx.doi.org/10.1016/ j.jpainsymman.2009.04.007

38. Bauer M, Ahmed S, Benedetti A, Greenaway C, Lalli M, Leavens A, et al. The impact of tuberculosis on health utility: a longitudinal cohort study. Qual Life Res. 2015;24:1337-49. http://dx.doi.org/10.1007/s11136-014-0858-6

39. Tagmouti S, Slater M, Benedetti A, Kik SV, Banaei N, Cattamanchi A, et al. Reproducibility of interferon gamma (IFN- $\gamma$ ) release assays. a systematic review. Ann Am Thorac Soc. 2014; 11:1267-76. http://dx.doi.org/10.1513/AnnalsATS.201405-188OC

40. Pouchot J, Grasland A, Collet C, Coste J, Esdaile JM,

Vinceneux P. Reliability of tuberculin skin test measurement. Ann Intern Med. 1997;126:210-4. http://dx.doi.org/10.7326/ 0003-4819-126-3-199702010-00005

41. Douglas P, Posey DL, Zenner D, Robson J, Abubakar I, Giovinazzo G. Capacity strengthening through pre-migration tuberculosis screening programmes: IRHWG experiences. Int J Tuberc Lung Dis. 2017;21:737-45. http://dx.doi.org/10.5588/ijtld.17.0019

42. Schwartzman K, Menzies D. Tuberculosis screening of immigrants to low-prevalence countries: a cost-effectiveness analysis. Am J Respir Crit Care Med. 2000;161:780-9. http://dx.doi.org/10.1164/ ajrccm.161.3.9902005

43. Schwartzman K, Oxlade O, Barr RG, Grimard F, Acosta I, Baez J, et al. Domestic returns from investment in the control of tuberculosis in other countries. N Engl J Med. 2005;353:1008-20. http://dx.doi.org/10.1056/NEJMsa043194

44. Sterling TR, Villarino ME, Borisov AS, Shang N, Gordin F, Bliven-Sizemore E, et al.; TB Trials Consortium PREVENT TB Study Team. Three months of rifapentine and isoniazid for latent tuberculosis infection. N Engl J Med. 2011;365:2155-66. http://dx.doi.org/10.1056/NEJMoa1104875

45. Byford S, Raftery J. Perspectives in economic evaluation. BMJ. 1998;316:1529-30. http://dx.doi.org/10.1136/bmj.316.7143.1529

46. Comstock GW. Frost revisited: the modern epidemiology of tuberculosis. Am J Epidemiol. 1975;101:363-82. http://dx.doi.org/10.1093/oxfordjournals.aje.a112105

Address for correspondence: Fawziah Marra, University of British Columbia, 2405 Wesbrook Mall, Vancouver, BC V6T 1Z3, Canada; email: fawziah@mail.ubc.ca

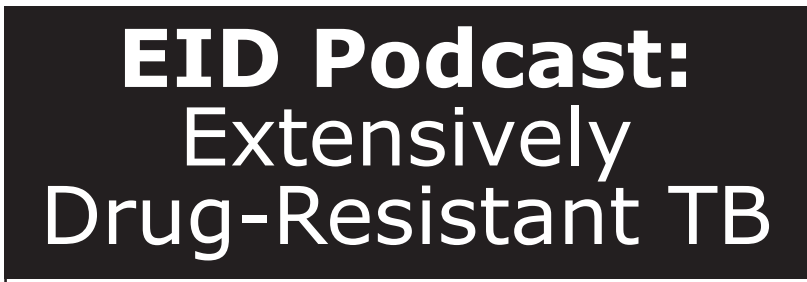

Tuberculosis (TB) remains a major cause of illness and death in the 21 st century. There were an estimated 9.6 million incident cases worldwide in 2014. In addition, an estimated $3.3 \%$ of new cases and $20 \%$ of retreatment cases are multidrug-resistant TB (MDR TB), which is defined as TB resistant to at least rifampin and isoniazid, the 2 most powerful first-line drugs. This resistance threatens global TB control efforts. MDR TB patients need access to treatment, require longer treatment with toxic medications, and have a lower probability of cure.

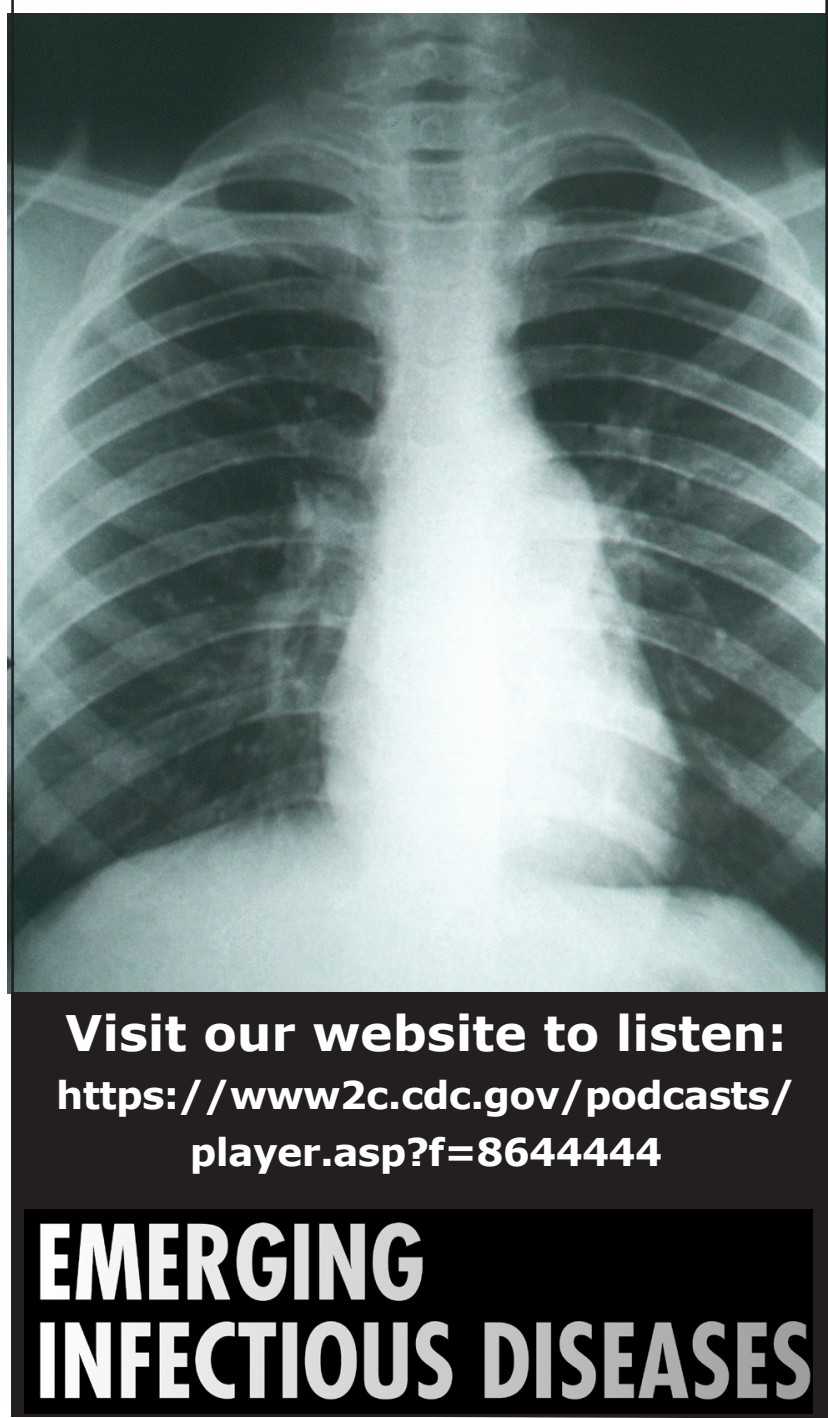

